\title{
An alternative construction for the Type-II defect matrix for the sshG
}

\author{
A.R. Aguirre, J.F. Gomes, A.L. Retore, N.I. Spano, and A.H. Zimerman
}

\begin{abstract}
In this paper we construct a Type-II defect (super) matrix for the supersymmetric sinh-Gordon model as a product of two Type-I defect (super) matrices. We also show that the resulting defect matrix corresponds to a fused defect.
\end{abstract}

\section{Introduction}

Integrable classical field theories with defects and its connection with Type-I and Type-II Bäcklund transformations (BT) has been widely studied in recent years by using mainly the Lagrangian formalism and the defect matrix approach [1]- [11]. The classical integrability is ensured by the derivation of modified higher order conserved quantities, which requires explicit solutions for the corresponding defect matrices.

On the other hand, the supersymmetric extensions for Liouville and sinh-Gordon (sshG) models with Type-I and Type-II defects has been also discussed in [12]- [15], and their associated defect matrices constructed.

More recently, it has been proposed in [18] that Type-II defect matrices could be constructed as a product of two Type-I defect matrices. This proposal was checked for the bosonic case of the mKdV hierarchy.

The aim of this paper is to verify this proposal for the sshG model and show that the resulting defect matrix corresponds to a fused defect.

A.R. Aguirre

Instituto de Física e Química, Universidade Federal de Itajubá - IFQ/UNIFEI, Av. BPS 1303, 37500-903, Itajubá, MG, Brasil. e-mail: alexis.roaaguirre@unifei.edu.br

J.F. Gomes, A.L. Retore, N.I. Spano, and A.H. Zimerman

Instituto de Física Teórica - IFT/UNESP, Rua Dr. Bento Teobaldo Ferraz 271, Bloco II, 01140070, São Paulo, Brasil. e-mail: jfg@ift.unesp.br, retore@ ift.unesp.br, natyspano@unesp.br, zimerman@ift.unesp.br 


\section{Type-I and Type II defect formulation}

The Lagrangian density describing the $N=1 \mathrm{sshG}$ model with Type-I defects located at $x=x_{1}$ can be written as follows,

$$
\mathscr{L}=\theta\left(x_{1}-x\right) \mathscr{L}_{1}+\delta\left(x-x_{1}\right) \mathscr{L}_{D_{1}}+\theta\left(x-x_{1}\right) \mathscr{L}_{0}
$$

with

$$
\begin{aligned}
\mathscr{L}_{p}= & \frac{1}{2}\left(\partial_{x} \phi_{p}\right)^{2}-\frac{1}{2}\left(\partial_{t} \phi_{p}\right)^{2}+i \psi_{p}\left(\partial_{x}+\partial_{t}\right) \psi_{p}-i \bar{\psi}_{p}\left(\partial_{x}-\partial_{t}\right) \bar{\psi}_{p} \\
& +4\left[\cosh \left(2 \phi_{p}\right)-1\right]-8 i \bar{\psi}_{p} \psi_{p} \cosh \phi_{p} \\
\mathscr{L}_{D_{1}}= & \frac{1}{2}\left(\phi_{0} \partial_{t} \phi_{1}-\phi_{1} \partial_{t} \phi_{0}\right)-i \psi_{1} \psi_{0}-i \bar{\psi}_{1} \bar{\psi}_{0}+2 i g_{1} \partial_{t} g_{1}+B_{0}^{(1)}+B_{1}^{(1)}
\end{aligned}
$$

where $\phi_{p}$ is a real scalar field, and $\psi_{p}, \bar{\psi}_{p}$ are the components of a Majorana spinor field in the regions $x>x_{1}(p=0)$ and $x<x_{1}(p=1)$ respectively, and $g_{1}$ an auxiliary fermionic field defined at the defect point. The defect potentials are given by,

$$
\begin{aligned}
& B_{0}^{(1)}=2 \sigma_{1} \cosh \left(\phi_{0}+\phi_{1}\right)+\frac{2}{\sigma_{1}} \cosh \left(\phi_{0}-\phi_{1}\right) \\
& B_{1}^{(1)}=2 i \sqrt{2} g_{1}\left[\sqrt{\sigma_{1}} \cosh \left(\frac{\phi_{0}+\phi_{1}}{2}\right)\left(\bar{\psi}_{0}+\bar{\psi}_{1}\right)+\frac{1}{\sqrt{\sigma_{1}}} \cosh \left(\frac{\phi_{0}-\phi_{1}}{2}\right)\left(\psi_{0}-\psi_{1}\right)\right]
\end{aligned}
$$

where $\sigma_{1}$ represent the Bäcklund parameter. Besides the bulk field equations, we get the following defect equations at $x=x_{1}$,

$$
\begin{aligned}
\partial_{t} \phi_{0}-\partial_{x} \phi_{1} & =2 \sigma_{1} \sinh \left(\phi_{0}+\phi_{1}\right)-\frac{2}{\sigma_{1}} \sinh \left(\phi_{0}-\phi_{1}\right) \\
& +\sqrt{2 \sigma_{1}} i_{1}\left[\sinh \left(\frac{\phi_{0}+\phi_{1}}{2}\right)\left(\bar{\psi}_{0}+\bar{\psi}_{1}\right)-\frac{1}{\sigma_{1}} \sinh \left(\frac{\phi_{0}-\phi_{1}}{2}\right)\left(\psi_{0}-\psi_{1}\right)\right] \\
\partial_{x} \phi_{0}-\partial_{t} \phi_{1} & =2 \sigma_{1} \sinh \left(\phi_{0}+\phi_{1}\right)+\frac{2}{\sigma_{1}} \sinh \left(\phi_{0}-\phi_{1}\right) \\
& +\sqrt{2 \sigma_{1}} i g_{1}\left[\sinh \left(\frac{\phi_{0}+\phi_{1}}{2}\right)\left(\bar{\psi}_{0}+\bar{\psi}_{1}\right)+\frac{1}{\sigma_{1}} \sinh \left(\frac{\phi_{0}-\phi_{1}}{2}\right)\left(\psi_{0}-\psi_{1}\right)\right] \\
\psi_{0}+\psi_{1} & =2 \sqrt{\frac{2}{\sigma_{1}}} \cosh \left(\frac{\phi_{0}-\phi_{1}}{2}\right) g_{1}, \\
\bar{\psi}_{0}-\bar{\psi}_{1} & =-2 \sqrt{2 \sigma_{1}} \cosh \left(\frac{\phi_{0}+\phi_{1}}{2}\right) g_{1}, \\
\partial_{t} g_{1} & =\sqrt{\frac{\sigma_{1}}{2}}\left[\frac{1}{\sigma_{1}} \cosh \left(\frac{\phi_{0}-\phi_{1}}{2}\right)\left(\psi_{1}-\psi_{0}\right)-\cosh \left(\frac{\phi_{0}+\phi_{1}}{2}\right)\left(\bar{\psi}_{0}+\bar{\psi}_{1}\right)\right] .
\end{aligned}
$$

These defect conditions preserve the integrability of the system after considering defect contributions to the conserved quantities [14]. The generating function for an infinite set of modified conserved quantities depends on the existence of the defect 
matrix $K_{1}$ connecting two field configurations, namely $\Psi^{(0)}=K_{1} \Psi^{(1)}$, satisfying the following equations,

$$
\partial_{ \pm} K_{1}=K_{1} A_{ \pm}^{(1)}-A_{ \pm}^{(0)} K_{1}
$$

where $\partial_{ \pm}=\frac{1}{2}\left(\partial_{x} \pm \partial_{t}\right), \lambda$ is a spectral parameter, and $\Psi^{(p)}$ are vector-valued fields satisfying the associated auxiliary linear problem, $\partial_{ \pm} \Psi^{(p)}=-A_{ \pm}^{(p)} \Psi^{(p)}$. The Lax pair $A_{ \pm}^{(p)}$ are $3 \times 3$ graded matrices valued in the $s l(2,1)$ Lie superalgebra, which can be written in the following form,

$$
\begin{aligned}
A_{+}^{(p)} & =\left(\begin{array}{cc|c}
\lambda^{1 / 2}-\partial_{+} \phi_{p} & -1 & \sqrt{i} \bar{\psi}_{p} \\
-\lambda & \lambda^{1 / 2}+\partial_{+} \phi_{p} & \lambda^{1 / 2} \sqrt{i} \bar{\psi}_{p} \\
\hline \lambda^{1 / 2} \sqrt{i} \bar{\psi}_{p} & \sqrt{i} \bar{\psi}_{p} & 2 \lambda^{1 / 2}
\end{array}\right), \\
A_{-}^{(p)} & =\left(\begin{array}{cc|c}
\lambda^{-1 / 2} & -\lambda^{-1} e^{2 \phi_{p}} & \lambda^{-1 / 2} \sqrt{i} \psi_{p} e^{\phi_{p}} \\
-e^{-2 \phi_{p}} & \lambda^{-1 / 2} & \sqrt{i} \psi_{p} e^{-\phi_{p}} \\
\hline-\sqrt{i} \psi_{p} e^{-\phi_{p}}-\sqrt{i} \lambda^{-1 / 2} \psi_{p} e^{\phi_{p}} & 2 \lambda^{-1 / 2}
\end{array}\right) .
\end{aligned}
$$

Therefore, we find that a suitable solution for the type-I defect matrix $\mathrm{K}$ can be written in the following explicit form [14],

$$
K_{1}=c_{1} \lambda^{1 / 2}\left(\begin{array}{cc|c}
1 & \frac{\sigma_{1}}{\lambda} e^{\phi_{1}+\phi_{0}} & -\sqrt{\frac{2 i \sigma_{1}}{\lambda} e^{\frac{\phi_{1}+\phi_{0}}{2}} g_{1}} \\
\hline \sigma_{1} e^{-\left(\phi_{1}+\phi_{0}\right)} & 1 & -\sqrt{2 i \sigma_{1}} e^{-\frac{\left(\phi_{1}+\phi_{0}\right)}{2}} g_{1} \\
\hline \sqrt{2 i \sigma_{1}} e^{-\frac{\left(\phi_{1}+\phi_{0}\right)}{2}} g_{1} & \sqrt{\frac{2 i \sigma_{1}}{\lambda}} e^{\frac{\left(\phi_{1}+\phi_{0}\right)}{2}} g_{1} & 1-\frac{\sigma_{1}}{\lambda^{1 / 2}}
\end{array}\right),
$$

where $c_{1}$ is a free constant parameter.

Now, the Type-II defect for the $N=1 \mathrm{sshG}$ model can be constructed by considering initially a two-defects system of the Type-I at different points, and then fusing them to the same point by taking a limit in the Lagrangian density [15]- [17]. Let us consider one of the defects placed at $x=x_{1}$ and the other at $x=x_{2}$. The Lagrangian density for this system can be written as follows,

$$
\begin{aligned}
\mathscr{L}= & \theta\left(x_{1}-x\right) \mathscr{L}_{1}+\delta\left(x-x_{1}\right) \mathscr{L}_{D_{1}}+\theta\left(x-x_{1}\right) \theta\left(x_{2}-x\right) \mathscr{L}_{0} \\
& +\delta\left(x-x_{2}\right) \mathscr{L}_{D_{2}}+\theta\left(x-x_{2}\right) \mathscr{L}_{2},
\end{aligned}
$$

where $\mathscr{L}_{p}$, with $p=0,1,2$, is given by eq. (2), and the two type-I defect Lagrangian densities at $x=x_{k}, k=1,2$, are given by eq. (3). Now, we have two auxiliary fermionic fields $g_{k}$, and two free parameters $\sigma_{k}$, with $k=1,2$, defined at the defect positions, respectively. At Lagrangian level, the fusing of defects can be performed by taking the limit $x_{2} \rightarrow x_{1}$. After some manipulations, it was shown that the fused defect is equivalent to a type-II defect [15], and takes the following form 
with $\phi_{ \pm}=\phi_{1} \pm \phi_{2}, \psi_{ \pm}=\psi_{1} \pm \psi_{2}$, and $B=B_{0}^{(+)}+B_{0}^{(-)}+B_{1}^{(+)}+B_{1}^{(-)}$the defect potentials,

$$
\begin{aligned}
B_{0}^{(+)}= & m \sigma\left[e^{\left(\phi_{+}-\lambda_{0}\right)}+e^{-\left(\phi_{+}-\lambda_{0}\right)}\left(\sinh ^{2}\left(\frac{\phi_{-}}{2}\right)+\cosh ^{2} \tau\right)\right] \\
B_{0}^{(-)}= & \frac{m}{\sigma}\left[e^{-\lambda_{0}}+e^{\lambda_{0}}\left(\sinh ^{2}\left(\frac{\phi_{-}}{2}\right)+\cosh ^{2} \tau\right)\right] \\
B_{1}^{(+)}= & -i \sqrt{m \sigma}\left[\left(e^{\frac{\left(\phi_{+}-\lambda_{0}\right)}{2}}+e^{-\frac{\left(\phi_{+}-\lambda_{0}\right)}{2}} \cosh \tau\right) \bar{\psi}_{+} f_{1}+e^{-\frac{\left(\phi_{+}-\lambda_{0}\right)}{2}} \sinh \left(\frac{\phi_{-}}{2}\right) \bar{\psi}_{+} \tilde{f}_{1}\right] \\
& +i m \sigma\left(1+e^{-\left(\phi_{+}-\lambda_{0}\right)} \cosh \tau\right) \cosh \left(\frac{\phi_{-}}{2}\right) f_{1} \tilde{f}_{1}, \\
B_{1}^{(-)}= & -i \sqrt{\frac{m}{\sigma}}\left[\left(e^{-\frac{\lambda_{0}}{2}}+e^{\frac{\lambda_{0}}{2}} \cosh \tau\right) \psi_{+} \tilde{f}_{1}-e^{\frac{\lambda_{0}}{2}} \sinh \left(\frac{\phi_{-}}{2}\right) \psi_{+} f_{1}\right] \\
& +\frac{i m}{\sigma}\left(1+e^{\lambda_{0}} \cosh \tau\right) \cosh \left(\frac{\phi_{-}}{2}\right) f_{1} \tilde{f}_{1}
\end{aligned}
$$

where it has been used $\sigma_{1}=\sigma e^{-\tau}, \sigma_{2}=\sigma e^{\tau}$, and the reparametrizations

$$
\begin{aligned}
& \phi_{0} \rightarrow-\lambda_{0}+\frac{\phi_{+}}{2}-\ln \left[\cosh \left(\frac{\phi_{-}}{2}-\tau\right)\right]-\frac{i}{2} \operatorname{sech}\left(\frac{\phi_{-}}{2}-\tau\right) f_{1} \tilde{f}_{1} \\
& f_{1}=\mu_{+} g_{2}+\mu_{-} g_{1}, \quad \tilde{f}_{1}=\mu_{-} g_{2}-\mu_{+} g_{1}, \quad \mu_{ \pm}=\left[\frac{1+e^{ \pm\left(\phi_{-}-2 \tau\right)}}{2}\right]^{-\frac{1}{2}}
\end{aligned}
$$

From the above defect Lagrangian we can write the defect conditions at $x_{1}=x_{2}$,

$$
\begin{aligned}
\left(\partial_{x}-\partial_{t}\right) \phi_{+}= & \partial_{t} \lambda_{0}-m\left[\sigma e^{-\left(\phi_{+}-\lambda_{0}\right)}+\frac{1}{\sigma} e^{\lambda_{0}}\right] \sinh \phi_{-}-i m\left(\sigma+\frac{1}{\sigma}\right) \sinh \left(\frac{\phi_{-}}{2}\right) f_{1} \tilde{f}_{1} \\
& +i \sqrt{m \sigma} e^{-\frac{\left(\phi_{+}-\lambda_{0}\right)}{2}} \cosh \left(\frac{\phi_{-}}{2}\right) \bar{\psi}_{+} \tilde{f}_{1}-i \sqrt{\frac{m}{\sigma}} e^{\frac{\lambda_{0}}{2}} \cosh \left(\frac{\phi_{-}}{2}\right) \psi_{+} f_{1} \\
& -i m\left[\sigma e^{-\left(\phi_{+}-\lambda_{0}\right)}+\frac{1}{\sigma} e^{\lambda_{0}}\right] \cosh \tau \sinh \left(\frac{\phi_{-}}{2}\right) f_{1} \tilde{f}_{1} \\
\left(\partial_{x}+\partial_{t}\right) \phi_{-}= & 2 m \sigma\left[e^{-\left(\phi_{+}-\lambda_{0}\right)}\left(\sinh ^{2}\left(\frac{\phi_{-}}{2}\right)+\cosh ^{2} \tau\right)-e^{\left(\phi_{+}-\lambda_{0}\right)}\right] \\
& +i \sqrt{m \sigma}\left(e^{\frac{\left(\phi_{+}-\lambda_{0}\right)}{2}}-e^{-\frac{\left(\phi_{+}-\lambda_{0}\right)}{2}} \cosh \tau\right) \bar{\psi}_{+} f_{1} \\
& -i \sqrt{m \sigma} e^{-\frac{\left(\phi_{+}-\lambda_{0}\right)}{2}} \sinh \left(\frac{\phi_{-}}{2}\right) \bar{\psi}_{+} \tilde{f}_{1} \\
& +2 i m \sigma e^{-\left(\phi_{+}-\lambda_{0}\right)} \cosh \tau \cosh \left(\frac{\phi_{-}}{2}\right) f_{1} \tilde{f}_{1}
\end{aligned}
$$




$$
\begin{aligned}
\left(\partial_{x}-\partial_{t}\right) \phi_{-}= & \frac{2 m}{\sigma}\left[e^{-\lambda_{0}}-e^{\lambda_{0}}\left(\sinh ^{2}\left(\frac{\phi_{-}}{2}\right)+\cosh ^{2} \tau\right)\right] \\
& -i \sqrt{\frac{m}{\sigma}}\left[\left(e^{-\frac{\lambda_{0}}{2}}-e^{\frac{\lambda_{0}}{2}} \cosh \tau\right) \psi_{+} \tilde{f}_{1}+e^{\frac{\lambda_{0}}{2}} \sinh \left(\frac{\phi_{-}}{2}\right) \psi_{+} f_{1}\right] \\
& -\frac{2 i m}{\sigma} e^{\lambda_{0}} \cosh \tau \cosh \left(\frac{\phi_{-}}{2}\right) f_{1} \tilde{f}_{1}, \\
\psi_{-}= & \sqrt{\frac{m}{\sigma}}\left[e^{\frac{\lambda_{0}}{2}} \sinh \left(\frac{\phi_{-}}{2}\right) f_{1}-\left(e^{-\frac{\lambda_{0}}{2}}+e^{\frac{\lambda_{0}}{2}} \cosh \tau\right) \tilde{f}_{1}\right], \\
\bar{\psi}_{-}= & \sqrt{m \sigma}\left[\left(e^{\frac{\left(\phi_{+}-\lambda_{0}\right)}{2}}+e^{-\frac{\left(\phi_{+}-\lambda_{0}\right)}{2}} \cosh \tau\right) f_{1}+e^{-\frac{\left(\phi_{+}-\lambda_{0}\right)}{2}} \sinh \left(\frac{\phi_{-}}{2}\right) \tilde{f}_{1}\right],(26) \\
\partial_{t} f_{1}= & -\frac{\sqrt{m \sigma}}{2}\left(e^{\frac{\left(\phi_{+}-\lambda_{0}\right)}{2}}+e^{-\frac{\left(\phi_{+}-\lambda_{0}\right)}{2}} \cosh \tau\right) \bar{\psi}_{+}+\frac{1}{2} \sqrt{\frac{m}{\sigma}} e^{\frac{\lambda_{0}}{2}} \sinh \left(\frac{\phi_{-}}{2}\right) \psi_{+} \\
& -\frac{m}{2}\left[\left(\sigma+\frac{1}{\sigma}\right)+\left(\sigma e^{-\left(\phi_{+}-\lambda_{0}\right)}+\frac{1}{\sigma} e^{\lambda_{0}}\right) \cosh \tau\right] \cosh \left(\frac{\phi_{-}}{2}\right) \tilde{f}_{1}, \quad(27) \\
\partial_{t} \tilde{f}_{1}= & -\frac{\sqrt{m \sigma}}{2} e^{-\frac{\left(\phi_{+}-\lambda_{0}\right)}{2}} \sinh \left(\frac{\phi_{-}}{2}\right) \bar{\psi}_{+}-\frac{1}{2} \sqrt{\frac{m}{\sigma}}\left(e^{-\frac{\lambda_{0}}{2}}+e^{\frac{\lambda_{0}}{2}} \cosh \tau\right) \psi_{+} \\
& +\frac{m}{2}\left[\left(\sigma+\frac{1}{\sigma}\right)+\left(\sigma e^{-\left(\phi_{+}-\lambda_{0}\right)}+\frac{1}{\sigma} e^{\lambda_{0}}\right) \cosh \tau\right] \cosh \left(\frac{\phi_{-}}{2}\right) f_{1} . \quad(28)
\end{aligned}
$$

In order to derive the associated Type-II defect super-matrix for the model, we propose [18] to construct it as a product of two Type-I defect matrices, such that

$$
\Psi^{(2)}=K_{1}\left(\sigma_{2}\right) \Psi^{(0)}=K_{1}\left(\sigma_{2}\right) K_{1}\left(\sigma_{1}\right) \Psi^{(1)}=K_{2}(\sigma, \tau) \Psi^{(1)},
$$

where $K_{2}(\sigma, \tau)=K_{1}\left(\sigma_{2}\right) K_{1}\left(\sigma_{1}\right)$. Therefore, by a direct computation we find that the components $k_{i j}$ of the fused defect matrix $K_{2}$ are given by,

$$
\begin{aligned}
k_{11}= & c\left(\lambda+\sigma^{2} e^{-\phi_{-}}+2 i \sigma e^{-\frac{\phi_{-}}{2}}\left(g_{1} g_{2}\right) \lambda^{1 / 2}\right), \\
k_{12}= & c \sigma e^{\phi_{0}}\left(e^{\left(\phi_{1}-\tau\right)}+e^{\left(\phi_{2}+\tau\right)}+2 i e^{\frac{\phi_{+}}{2}}\left(g_{1} g_{2}\right)\right), \\
k_{13}= & -c \sigma \sqrt{2 i \sigma} e^{\frac{\phi_{0}}{2}}\left(e^{\phi_{2}-\frac{\left(\phi_{1}-\tau\right)}{2}} g_{1}-e^{\frac{\left(\phi_{2}-\tau\right)}{2}} g_{2}\right) \\
& -c \sqrt{2 i \sigma} \lambda^{1 / 2} e^{\frac{\phi_{0}}{2}}\left(e^{\frac{\left(\phi_{1}-\tau\right)}{2}} g_{1}+e^{\frac{\left(\phi_{2}+\tau\right)}{2}} g_{2}\right) \\
k_{21}= & c \sigma e^{-\phi_{0}}\left(e^{-\left(\phi_{1}+\tau\right)}+e^{-\left(\phi_{2}-\tau\right)}+2 i e^{-\frac{\phi_{+}}{2}} g_{1} g_{2}\right), \\
k_{22}= & c\left(\lambda+\sigma^{2} e^{\phi_{-}}+2 i \sigma e^{-\frac{\phi_{-}}{2}} g_{1} g_{2}\right), \\
k_{23}= & -c \sqrt{2 i \sigma} \lambda e^{-\frac{\phi_{0}}{2}}\left(g_{1} e^{-\frac{\left(\phi_{1}+\tau\right)}{2}}+g_{2} e^{-\frac{\left(\phi_{2}-\tau\right)}{2}}\right) \\
& +c \sigma \sqrt{2 i \sigma} \lambda^{1 / 2} e^{-\frac{\phi_{0}}{2}}\left(g_{2} e^{-\frac{\left(\phi_{2}+\tau\right)}{2}}-g_{1} e^{\frac{\left(\phi_{1}+\tau\right)}{2}-\phi_{2}}\right),
\end{aligned}
$$




$$
\begin{aligned}
k_{31}= & c \sqrt{2 i \sigma} \lambda e^{-\frac{\phi_{0}}{2}}\left(g_{1} e^{-\frac{\left(\phi_{1}+\tau\right)}{2}}+g_{2} e^{-\frac{\left(\phi_{2}-\tau\right)}{2}}\right) \\
& +c \sigma \sqrt{2 i \sigma} \lambda^{1 / 2} e^{-\frac{\phi_{0}}{2}}\left(g_{2} e^{\frac{\left(\phi_{2}-\tau\right)}{2}-\phi_{1}}-g_{1} e^{\frac{\left(\phi_{1}+\tau\right)}{2}}\right), \\
k_{32}= & c \sigma \sqrt{2 i \sigma} e^{\frac{\phi_{0}}{2}}\left(g_{2} e^{-\frac{\left(\phi_{2}+\tau\right)}{2}+\phi_{1}}-g_{1} e^{\frac{\left(\phi_{1}+\tau\right)}{2}}\right) \\
& +c \sqrt{2 i \sigma} \lambda^{1 / 2} e^{\frac{\phi_{0}}{2}}\left(e^{\frac{\left(\phi_{1}-\tau\right)}{2}} g_{1}+e^{\frac{\left(\phi_{2}+\tau\right)}{2}} g_{2}\right) \\
k_{33}= & c\left(\lambda+\sigma^{2}-2 \sigma \lambda^{1 / 2}\left(\cosh (\tau)-2 i g_{1} g_{2} \cosh \left(\frac{\phi_{-}}{2}\right)\right),\right.
\end{aligned}
$$

where $c=c_{1} c_{2}$. By straightforward comparison with eq. (A.80)-(A.89) in [15], it is not difficult to see that the fused defect matrix derived as product of two type-I defect matrices is equivalent (up to $\lambda^{1 / 2}$ ) to the type-II defect matrix previously found in [15], after reparametrazing the auxiliary fields given as in eqs. (20) and (21).

Acknowledgements The authors would like to thank the organizers of the colloquium ICGTMP - Group 31 for the opportunity to present our work. ALR would like to thank FAPESP for the financial support under the process 2015/00025-9. JFG would like to thank FAPESP and CNPq for the financial support. NIS and AHZ would like to thank CNPq for the financial support.

\section{References}

1. P. Bowcock, E. Corrigan and C. Zambon, Int. J. Mod. Phys. A 19S2 (2004) 82; JHEP 01 (2004) 056 ; JHEP 08 (2005) 23

2. V. Caudrelier, Int. J. Geom. Meth. Mod. Phys. 5 (2008) 1085

3. I. Habibullin and A. Kundu, Nucl. Phys. B 795 (2008) 549

4. J. Avan and A. Doikou, JHEP 01 (2012) 040

5. J. Avan and A. Doikou, JHEP 11 (2012) 008

6. V. Caudrelier and A. Kundu, JHEP 02 (2015) 088

7. V. Caudrelier, J. Phys. A 48 (2015) 195203

8. E. Corrigan and C. Zambon, J. Phys. A 42 (2009) 475203; J. Phys. A 43 (2010) 345201

9. A.R. Aguirre, T.R. Araujo, J.F. Gomes, and A.H. Zimerman, JHEP 12 (2011) 056

10. A.R. Aguirre, J. Phys. A 45 (2012) 205205

11. J. F. Gomes, A. L. Retore and A. H. Zimerman, J. Phys. A 48 (2015) 405203

12. A.R. Aguirre, J. Phys. Conf. Ser. 474 (2013) 012001.

13. J.F. Gomes, L.H. Ymai, and A.H. Zimerman, J. Phys. A 39 (2006) 7471

14. A.R. Aguirre, J.F. Gomes, N.I. Spano, A.H. Zimerman, JHEP 02 (2015) 175

15. A. R. Aguirre, J. F. Gomes, N. I. Spano and A. H. Zimerman, JHEP 06 (2015) 125

16. E. Corrigan and C. Zambon, J. Phys. A 43 (2010) 345201

17. C. Robertson, J. Phys. A 47 (2014) 185201

18. J.F.Gomes, A. L. Retore and A.H.Zimerman (submitted to publication) 